\title{
A Daily Diagnostic Multidisciplinary Meeting to Reduce Time to Definitive Diagnosis in the Context of Primary Bone and Soft Tissue Sarcoma
}

This article was published in the following Dove Press journal: Journal of Multidisciplinary Healthcare

Laura J Hartley $\mathbb{D}^{\prime}$

Scott Evans ${ }^{2}$

Mark A Davies ${ }^{2}$

Suzanne Kelly $\mathbb{D}^{2}$

Jonathan J Gregory ${ }^{2}$

'University Hospitals Birmingham NHS Foundation Trust, Birmingham, UK; ${ }^{2}$ The Royal Orthopaedic Hospital NHS Foundation Trust, Birmingham, UK
Correspondence: Laura J Hartley University Hospitals Birmingham NHS Foundation Trust, Birmingham, UK $\mathrm{Tel}+44$ I2I 4240000

Email I.hartley2@nhs.net
Background and Objectives: Cancer services are under increasing pressure to deliver waiting time targets. Our service has seen referral numbers increase to over 3000 per annum, with more than $80 \%$ coming from secondary care. In order to deliver a responsive service, the department has introduced a daily diagnostic multidisciplinary meeting (DMDT) with the aim being stratification of resources by directing rapid access to clinics and diagnostics to those felt to be at greatest risk of malignancy at the start of the pathway. It also aimed to improve communication with patients and referrers, consistency in decision making and deliver improved diagnostic turn-around times in a sustainable manner. An evaluation was undertaken to assess whether the introduction of the DMDT has improved the pathway, the primary endpoint being a reduction in time to definitive diagnosis (TTDD). Secondary endpoints included measurements of efficiency and whether there has been a reduction in variation in practice.

Methods: Retrospective access to a prospective database over a 1-month period before (2015) and after (2018) the intervention.

Results: The introduction of the DMDT has led to a reduction in TTDD ( 7 days). The service also has an added benefit in reducing average total patient miles travelled over the course of diagnosis by 22.68 miles.

Conclusion: The introduction of a diagnostic MDT at the start of the pathway does lead to an improvement in service efficiency and a reduction in TTDD.

Keywords: multidisciplinary, cancer, oncology, diagnosis, waiting times, sarcoma

\section{Synopsis}

An evaluation of our daily diagnostic multidisciplinary team meeting focusing on the improvement made to our diagnostic pathway and the overall impact on time to definitive diagnosis in the context of primary soft tissue and bone sarcoma.

\section{Introduction}

Multidisciplinary team (MDT) working has been the gold standard for care in all specialties since its introduction in the late 1990s. Before MDT meetings existed, there were large variations in clinical practice and the quality of reporting for radiological investigations and pathological specimens was poor. ${ }^{1}$ The CalmanHine report ${ }^{2}$ was commissioned by the Government in the hope that regular MDT meetings would enhance the practice of evidence-based medicine and personalise care whilst preventing individuals from treating patients outside of accepted 
standards. It also aimed to help reduce waiting times and improve the quality of care for patients with cancer; adhering to the age-old principle that many hands make light work. ${ }^{1,3}$ Over the last 20 years, the cancer landscape has altered dramatically due to the increasing complexity of cases, ageing population and the increasing range of treatment options available. Subsequently, cancer services in the UK are under heightened pressure to deliver on waiting times. For clinicians, increased demand often means less time spent per case, especially where centres are involved in diagnostic services. This poses a substantial patient safety and quality risk and has called into question the value of the MDT meeting. ${ }^{4}$

Despite the widespread adoption of the MDT approach, the Cancer Waiting Times Annual Report published by NHS England highlights that services in the UK are continually falling short of the expected $85 \%$ standard when delivering on 62-day referral to treatment waiting times for all cancers, achieving a maximum of $82.4 \%$ since $2015 .^{5-7}$ A recent analysis carried out by the British Medical Association suggested that waiting times have deteriorated since the publication of the Annual Report stating that between January and February of 2019 almost a quarter of patients have had to wait more than 2 months for their first treatment after an urgent referral from their GP - only $76.2 \%$ had their first treatment within the 62-day target, the worst performance against the set standard since it was introduced. ${ }^{8}$ Furthermore, cancer waiting times for diagnosis are set to decrease to 28 days as of April 2020, placing even more pressure on already stretched diagnostic services and making it even more important that our services are streamlined and efficient.

Several recent papers have highlighted that although MDT meetings are now an integral part of mainstream cancer pathways and adherence to their use is high, they are costly and do not function as effectively as they should. $^{2,9,10}$ The issues of cost and efficiency are highly relevant in the UK's financially challenged, resource scarce climate today. De Iso et al identified a need to improve the efficiency both in terms of cost and time spent on MDT meetings without compromising, or worse, losing the considerable benefits of regular meetings. ${ }^{9}$ It has been suggested that a large proportion of cases reaching MDT meetings today do not require this level of discussion and time better used on more complex cases is wasted. ${ }^{2,9-12}$ This is corroborated by a study from Cancer Research UK, which highlighted that 75\% MDT members agreed that some patients could be reviewed outside the MDT to enable more time for discussion of complex cases. ${ }^{11,13}$ Munro (2015) highlights that an average MDT will review between 15 and 30 patients per week, often repeating cases as more information and advice becomes available without progressing management in a meaningful way. Furthermore, the paper suggests that the MDT process consumes a disproportionate amount of the resources available for the care of patients with cancer. ${ }^{2}$

In light of these findings, the Cancer Transformation Board and the Department of Health carried out a project that aimed to reform MDT meetings to make them more effective in response to the increased demand. The project identified the need for a number of changes, including streamlining the current MDTs to make them more efficient and the need to return ownership and responsibility to individuals by giving them permission to make shared decisions with select patients without necessarily seeking approval from the MDT. ${ }^{14,15}$ Recommendation 38 from the Independent Cancer Taskforce Report agreed that the need to focus specialist time in MDT meetings on cases which do not follow established clinical pathways is a priority. ${ }^{15}$

Primary soft tissue and bone sarcomas are rare mesenchymal tumours which can arise almost anywhere in the body. On average, a general practitioner may only see one case of primary sarcoma in the entirety of their career. Their relative rarity and inherent heterogeneity make it difficult to develop steadfast guidelines for their diagnosis and management. Currently, the diagnosis and management of primary sarcomas is guided by advice published by the British Sarcoma Group. ${ }^{16-18}$ The key recommendation from the guidelines states that any patient with red flag symptoms should receive urgent imaging appropriate to the suspected pathology (plain radiograph for bone, ultrasound for soft tissue) and if this was inconclusive or suggestive of malignancy, should be referred to and reviewed by a specialist sarcoma MDT for further assessment. ${ }^{16-18}$ In a review of national figures, the bone sarcoma incidence rate has remained stable between 1985 and 2009; however, the incidence rates of soft tissue sarcoma have risen by $26 \%$ between 1990 and $2007 .{ }^{19,20}$ At our institute, we have been running a weekly sarcoma MDT (WMDT) since 1993. The mean and maximum number of cases discussed at a single WMDT in 1993 were 15 and 31, respectively. In 2016, the mean and maximum number of cases reached 78 and 110 , respectively, representing an overall increase of $420 \%$ and $255 \%$ since $1993 .{ }^{21}$ Our department, as a leading centre for orthopaedic oncology worldwide, now receives over 3000 referrals per annum, 
with more than $80 \%$ of referrals coming from secondary care.

It was identified that this increase in WMDT discussions was unsustainable, from both a work force and a decision-making perspective. A review of our processes showed opportunities to reduce waste, waiting times and variation in clinical practice as well as improve safety by changing the model of referral ownership. It further highlighted opportunities to focus resources by case stratification, improve patient experience and at least meet, if not exceed the national target of 28 days for cancer diagnosis.

In order to deliver a responsive service, the department introduced a daily diagnostic MDT meeting (DMDT) in 2017 alongside its weekly sarcoma MDT (WMDT). The DMDT employs a true multidisciplinary approach, much in the same way as the WMDT, involving a consultant orthopaedic oncology surgeon, specialist consultant radiologist, clinical nurse specialist and an MDT coordinator as a minimum. It takes place daily (except for weekends) and is employed as a means of triaging the new referrals. All the findings, diagnostic decisions, and recommendations from the DMDT are directly entered into the patient's records held within a prospective oncology database in real time. The cases are triaged into malignancy likely, malignancy possible, malignancy unlikely and malignancy excluded. This is in stark contrast to the review of referrals employed before the introduction of the
DMDT, in which paper referrals were read by a single surgeon without direct input from a radiologist (Figures 1 and 2).

The objectives of the DMDT were to improve the pathway for our patients by stratifying resources and directing rapid access to diagnostics to those felt to be at greatest risk of malignancy at the start of the pathway. It also aimed to improve communication between clinicians and patients, to reduce the number of non-sarcoma cases discussed at the $\mathrm{WMDT}^{21}$ (thereby increasing cognitive bandwidth and time for discussion of complex cases) and to deliver clinical excellence in a patient centred, compassionate manner. Lastly, it aimed to provide consistency in decision making and deliver improved diagnostic turnaround times in a sustainable manner.

\section{Aims}

The aim of this evaluation is to assess whether the DMDT has improved the patient pathway, with the primary endpoints being a reduction in time to first management decision and a reduction in time to definitive diagnosis (TTDD). Secondary endpoints included measurements of the accuracy of the DMDT initial triage diagnosis compared to the definitive diagnosis on histology and the ability of the DMDT to discharge patients without the need for the patient to travel to our centre.

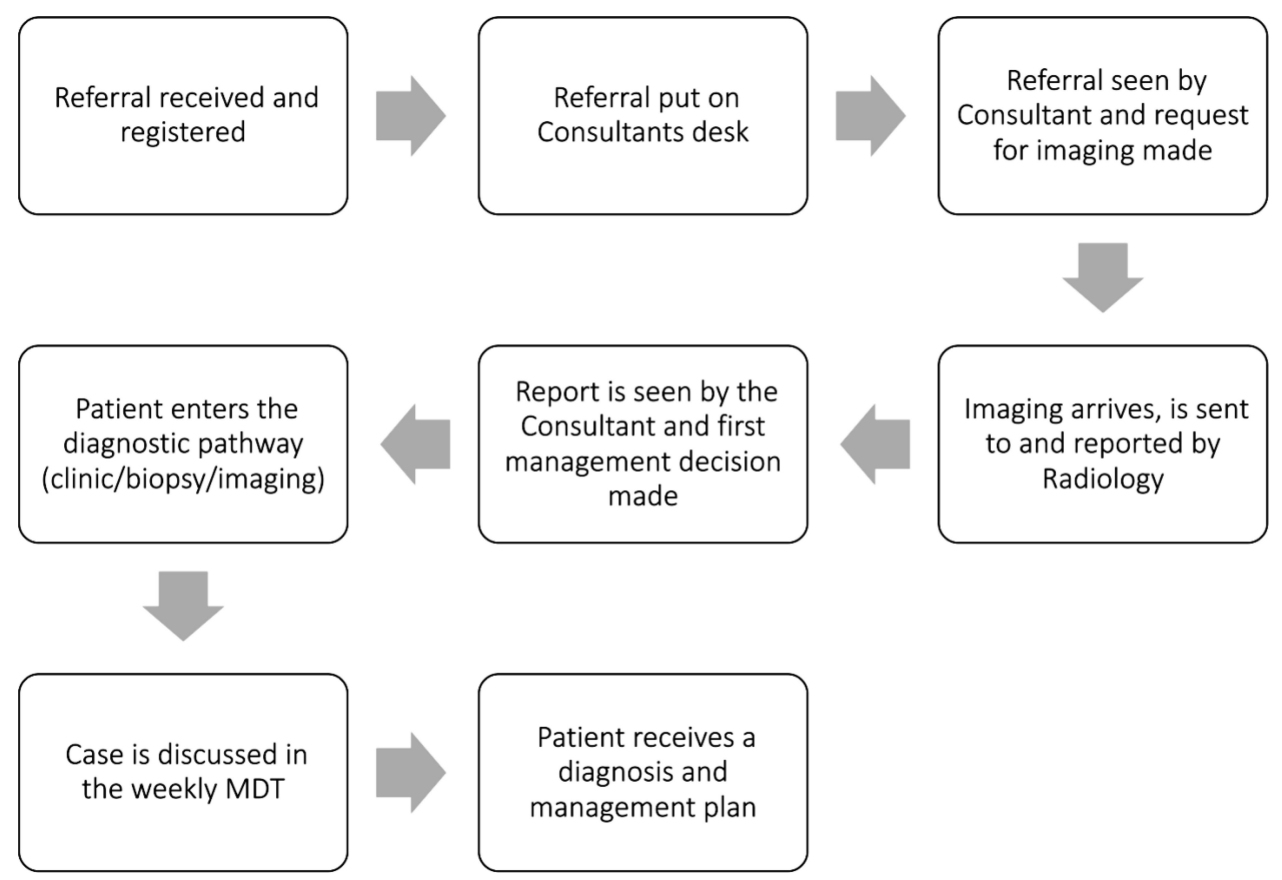

Figure I A depiction of the referral process in 2015 before the introduction of the DMDT; illustrating the steps taken to appropriately triage the referral before making the first management decision.

Abbreviation: MDT, multidisciplinary team meeting. 


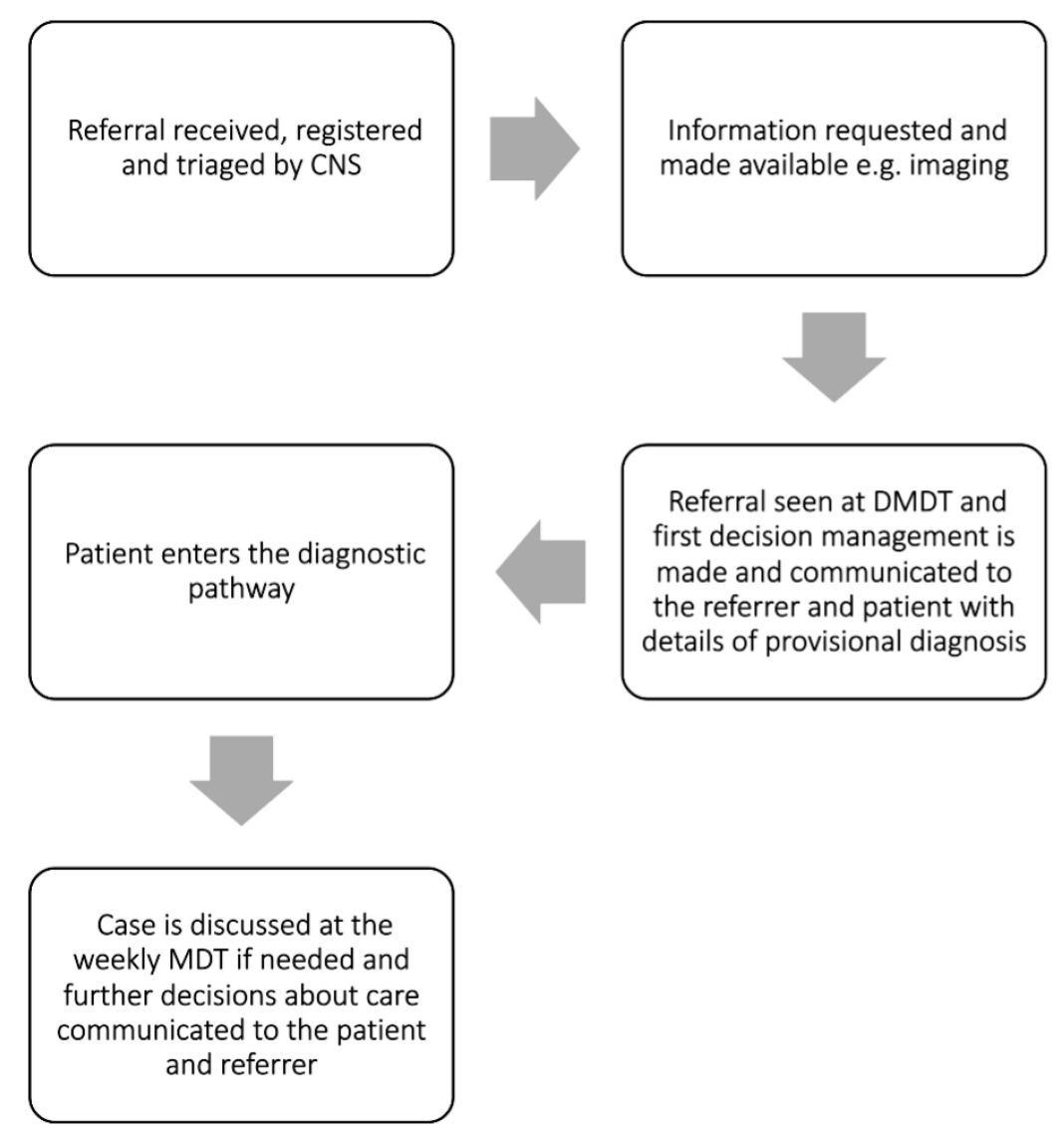

Figure 2 A depiction of the referral process in 2018 after the introduction of the DMDT; illustrating the steps taken to appropriately triage the referral and make a first management decision.

Abbreviations: MDT, multidisciplinary team meeting; CNS, clinical nurse specialist; DMDT, diagnostic multidisciplinary team meeting.

\section{Patients and Methods}

All patients referred for a suspected bone or soft tissue malignancy were identified by interrogating our prospectively maintained database. The search was conducted to include two time periods; the 1 st to the 31 st of January in both 2015 - before the introduction of the DMDT, and 2018 - after the introduction of the DMDT. Demographic data (Table 1) were collected along with time to triage the referral, time to a first management decision and provisional and final histological diagnosis (if a biopsy was performed) to determine overall accuracy of the DMDT triage.

Patients with a previous sarcoma diagnosis, cases designated "opinion only", patients who withdrew from the diagnostic pathway for any reason, patients without data on the systems, patients deceased before formal diagnosis, patients related to the researchers and private patients were excluded from the study to give a cohort whose data reflected the whole diagnostic pathway within the public healthcare system.
101 consecutive patients met the inclusion criteria and were selected for the 2015 cohort and 135 consecutive patients were selected for the 2018 cohort. For both cohorts, the following data was collected.

- Date the referral was received and registered on our system

- Date the referral was triaged/seen at DMDT

- The provisional diagnosis ascribed at DMDT/triage

- Date the first management decision was made and what the decision was

- Date the definitive diagnosis was made

For both cohorts, the total number of days and the number of working days between dates were calculated using online date counting software.

A Fisher's exact test was used to assess statistical significance of a $2 \times 2$ contingency table in the analysis of the primary endpoint. A p value was calculated with two tails. An alpha value of 0.05 was considered statistically significant. 
Table I Demographic Data for the 2015 and 2018 Cohorts

\begin{tabular}{|c|c|c|}
\hline & $2015, N=101$ & $2018, N=135$ \\
\hline Age & 95 Adults ${ }^{(94 \%)} 6$ Children $^{(6 \%)}$ & II6 Adults ${ }^{(86 \%)} 19$ Children $^{(14 \%)}$ \\
\hline Gender & 47 Females ${ }^{(46 \%)} 54$ Males $^{(54 \%)}$ & 67 Females ${ }^{(50 \%)} 68$ Males $^{(50 \%)}$ \\
\hline Type of Referral & $342 W^{(34 \%)} 63$ Routine $^{(62 \%)} 4$ Urgent $^{(4 \%)}$ & $292 W W W^{(21 \%)} 100$ Routine $^{(74 \%)} 6$ Urgent $^{(4 \%)}$ \\
\hline Referrer & $\begin{array}{l}69 \text { Other Hospital Consultant }{ }^{(68 \%)} \\
32 \mathrm{GP}^{(32 \%)}\end{array}$ & $\begin{array}{l}\text { I I } 2 \text { Other Hospital Consultant } \\
22 \mathrm{GP}^{(16 \%)} \\
\text { I Allied Healthcare Professional }{ }^{(1 \%)}\end{array}$ \\
\hline Pathway Used & $\begin{array}{l}29 \text { Suspected Primary Bone Sarcoma }{ }^{(29 \%)} \\
46 \text { Suspected Primary Soft Tissue Sarcoma }{ }^{(46 \%)} \\
26 \text { Other Bone Pathology }\end{array}$ & $\begin{array}{l}33 \text { Suspected Primary Bone Sarcoma }{ }^{(24 \%)} \\
53 \text { Suspected Primary Soft Tissue Sarcoma }{ }^{(40 \%)} \\
49 \text { Other Bone Pathology }{ }^{(36 \%)}\end{array}$ \\
\hline Definitive Diagnosis & $\begin{array}{l}4 \text { Primary Bone Sarcoma }{ }^{(4 \%)} \\
12 \text { Primary Soft Tissue Sarcoma } a^{(12 \%)} \\
3 \text { Haematological Malignancy }{ }^{(3 \%)} \\
5 \text { Metastatic Pathology }{ }^{(5 \%)} \\
77 \text { Benign (bone and soft tissue) Pathology }{ }^{(76 \%)}\end{array}$ & $\begin{array}{l}3 \text { Primary Bone Sarcoma }{ }^{(2 \%)} \\
\text { I I Primary Soft Tissue Sarcoma }{ }^{(8 \%)} \\
6 \text { Haematological Malignancy }{ }^{(4 \%)} \\
2 \text { I Metastatic Pathology }{ }^{(16 \%)} \\
94 \text { Benign (bone and soft tissue) Pathology }{ }^{(69 \%)}\end{array}$ \\
\hline
\end{tabular}

The service evaluation project on which this study was based was carried out with approval from our Clinical Audit and Effectiveness Team at our centre. Individual patient consent to access their medical records for the purpose of service evaluation was not required. The data were anonymised to maintain confidentiality and held securely in compliance with the Declaration of Helsinki.

\section{Results}

\section{Primary Endpoints}

\section{Time to Triage/DMDT and First Management} Decision

In 2015, on average, it took 2 total days for a referral to be triaged (median 1 day, range 1-22 days) and 11 total days to make a first management decision (median 8 days, range 1-124 days). In 2018, it took 3 total days to be seen at DMDT (median 3 days, range 1-22 days, an increase of 1 day $\mathrm{p}>0.05$ ) and an average of 5 total days to make a first management decision (median 3 days, range 1-109 days, a reduction of 6 total days $p>0.05$ ).

No change in working time to triage was observed between 2015 and 2018. A reduction of 5 working days was observed in time to first management decision (median 2 days, range $1-76$ days).

\section{Time to Definitive Diagnosis}

It took longer on average to diagnose the 2015 cohort than it did the 2018 cohort in terms of both total and working days.
Total Days to Definitive Diagnosis (TTDD)

For all cases in 2015, the mean TTDD was 41 days (median 29 days, range 1-158 days). For all cases in 2018, the mean TTDD was 34 days (median 28 days, range 2-152 days), a reduction of 7 days with the introduction of the DMDT ( $>0.05$ ).

\section{Working Days to Definitive Diagnosis (WTTD)}

For all cases in 2015, the mean WTTD was 29 days (median 21 days, range 1-109 days). For all cases in 2018, the mean WTTD was 24 days (median 21 days, range $2-105$ days), a reduction of 5 days with the introduction of the DMDT $(p>0.05)$.

\section{Secondary Endpoints}

\section{Provisional Diagnosis at Triage/DMDT}

No provisional diagnosis data were available for the 2015 cohort as the single surgeon review of the referrals was not triaged into the categories used at the DMDT i.e malignancy excluded, malignancy likely, malignancy possible and malignancy unlikely. For the 2018 cohort, provisional diagnosis was split into these four categories. Thirty cases were assigned "malignancy likely", 34 cases were subscribed "malignancy possible", 27 cases were assigned "malignancy unlikely" and 44 cases had malignancy excluded based on history and imaging alone. Further information regarding the provisional diagnosis can be found in Table 2. 
Table 2 Provisional Diagnosis at DMDT Data (2018)

\begin{tabular}{|l|l|l|}
\hline Group & Sub-Group & Number of Cases \\
\hline Malignancy Likely $(\mathrm{N}=30)$ & Sarcoma Malignancy (Bone/STS) & 15 \\
& Non-Sarcoma Malignancy (Metastatic Disease/Pathological Fracture) & 15 \\
Malignancy Possible ( $=34)$ & Sarcoma Malignancy (Bone/STS) & 32 \\
Malignancy Unlikely ( $=27)$ & Non-Sarcoma Malignancy (Metastatic Disease/Pathological Fracture) & 2 \\
Malignancy Excluded $(\mathrm{N}=44)$ & & \\
\hline
\end{tabular}

Four of the cases in the "malignancy likely" group were proven benign after biopsy, 8 were proven to be malignant and 18 were proven to be metastatic. Of the "malignancy possible" group, 4 were metastatic disease, 19 were benign and 11 were found to be malignant. Of the "malignancy unlikely" group, all 27 were found to be benign.

\section{Measurement of Accuracy of Triage/DMDT}

No provisional diagnoses were assigned in 2015 therefore we were unable to measure the accuracy of the single surgeon triage. Sensitivity of the DMDT was found to be $100 \%(95 \%$ CI $91.4 \%$ - 100\%). Specificity was found to be $54 \%$ (95\% CI 36.44\% - 57.39\%). Fifty cases ascribed a more sinister provisional diagnosis were downgraded to benign pathologies after biopsy. No cases ascribed a malignancy excluded provisional diagnosis were subsequently found to be malignant.

\section{Outcomes of the First Management Decision}

The outcomes of the first management decisions made in 2015 and 2018 are detailed in Tables 3 and 4 respectively. "Surgery" denotes excision of the lesion in theatre. "Clinic" denotes seen in a specialist orthopaedic oncology clinic by a consultant or registrar. "Biopsy" denotes the

Table 3 Outcomes of First Management Decision (2015)

\begin{tabular}{|l|l|l|l|}
\hline Clinic Only & $18 \%$ & Clinic + Scan + Biopsy & $1 \%$ \\
Biopsy Only & $21 \%$ & Scan + Biopsy & $10 \%$ \\
Scan Only & $7 \%$ & Discharge & $18 \%$ \\
Clinic + Scan & $16 \%$ & Palliative & $0 \%$ \\
Clinic + Biopsy & $6 \%$ & Surgery & $4 \%$ \\
\hline
\end{tabular}

Table 4 Outcomes of First Management Decision (2018)

\begin{tabular}{|l|l|l|l|}
\hline Clinic Only & $13 \%$ & Clinic + Scan + Biopsy & $11 \%$ \\
Biopsy Only & $3 \%$ & Scan + Biopsy & $1.5 \%$ \\
Scan Only & $1.5 \%$ & Discharge & $24 \%$ \\
Clinic + Scan & $19 \%$ & Palliative & $1.5 \%$ \\
Clinic + Biopsy & $24 \%$ & Surgery & $1.5 \%$ \\
\hline
\end{tabular}

procurement of a tissue sample by accepted means (CT or USS guided) without complete excision.

\section{Impact on Distance Travelled}

The accuracy of the DMDT means that in 2018, 24\% of patients are discharged at the point of triage removing the need to travel to our centre. Patients travelled on average a total of 172.94 miles over the course of their diagnosis in 2015 (median 80 miles, range 1-368 miles). With the introduction of the DMDT patients travelled on average a total of 150.26 miles over the course of their diagnosis (median 68 miles, range 1-489 miles). This represents a reduction in patient miles travelled of 22.68 miles $(p<0.05)$.

\section{Discussion}

\section{Statement of Principle Findings}

Our study has shown that the introduction of the DMDT has reduced our time to make a first management decision by 6 total days and our time to definitive diagnosis by 7 total days.

As a result of the introduction of the DMDT, 24\% of patients were discharged from our service without having to travel to our centre (Table 4), saving an average of 22.68 miles over the course of their diagnosis $(p<0.05)$ despite our catchment area expanding. The sensitivity and specificity of the DMDT further corroborate the accuracy of the triaging system. We have not seen any of the $24 \%$ of discharged patients (all with benign diagnoses) re-referred for further management or with a deteriorating clinical picture necessitating a change in diagnosis to a more sinister pathology.

We have also demonstrated an increase in the scheduling of multiple diagnostic techniques at the point of triage as a result of the introduction of the DMDT (11\% in 2018 compared to $1 \%$ in 2015 ) with a subsequent reduction in the scheduling of each of the single diagnostic services (biopsy only $21 \%$ in $2015-3 \%$ in 2018 , clinic only $18 \%$ in $2015-13 \%$ in 2018 and scan only $7 \%$ in $2015-1.5 \%$ in 
2018) highlighting our "one-stop shop" approach and hence, the increased efficiency of our service. We anticipate a further improvement in efficiency as the system matures. Further studies are ongoing to identify subsequent inefficiencies in the pathway which prevent us from reaching the national diagnostic target of 28 days.

\section{Relevance to Clinical Practice}

Subjectively, for the patient, nothing is more important than receiving a diagnosis and initiating management in an expedient and stress-free manner, ${ }^{22-27}$ especially in the context of cancer. The new Cancer Waiting Time Targets reflect this - the drive to diagnose patients and initiate treatment within 28 days puts added pressure on already overworked diagnostic centres but is inherently patient centred. Awaiting diagnosis and treatment has been cited as one of the most stressful experiences for patients in today's NHS, regardless of the nature of the potential diagnosis. ${ }^{27-30}$ The reduction in TTDD of 7 total days suggests that the use of a DMDT may be a reproducible way of reducing diagnostic turnaround times, helping us to meet the nationally mandated waiting time targets and reduce patient anxiety. Additionally, financial compensation for meeting targets incentivises services to accelerate diagnosis and treatment initiation. The financial implications of implementing such a service are outside the scope of this study and further research is needed.

We have not directly looked at the psychological impact of our DMDT on our patients beyond informal interviews where patients and carers have reported a high level of satisfaction with the service. However, we anticipate that it has had a positive effect. The DMDT affords us the ability to give patients an almost immediate provisional diagnosis and management plan as well as provide psychosocial support from Clinical Nurse Specialists. Furthermore, feedback to referring clinicians is delivered on the same day as the DMDT and our protocols dictate that routine referrals triaged as "malignancy likely" are upgraded onto a cancer pathway thereby further ensuring prompt progression along the clinical pathway, minimizing delays.

Aside from the psychological impact, we know from studies done in cancer settings in general that rapid diagnosis and treatment also has an impact on the clinical outcome. $^{20,22,28,29}$ Rarer cancers tend to be more advanced at presentation to diagnostic services and as such have poorer outcomes. ${ }^{31}$ Management of advanced cancers has also been shown to be more complex than those presenting early. ${ }^{28,29}$ Similarly, the overall mortality and morbidity of more advanced cancers has been shown to be higher. ${ }^{22,32}$ It, therefore, follows that the sooner the diagnosis is made, in our case -7 days earlier, the sooner treatment can be initiated and the better the potential outcome for the patient; however, we currently have no data to support this.

Despite incentives for meeting nationally imposed targets, our NHS is perpetually financially limited. Therefore, it is important that we use our limited resources in an efficient manner, directing them to those most in need. Our study has shown that nearly a quarter of patients are discharged from our service without the need to travel to our centre (Table 4). The "one-stop shop" approach to diagnostics (first proposed in Chang et al in $1998^{24}$ ) means faster access to clinic appointments, biopsies and scans for those felt to be at greatest risk at the start of the pathway. Additionally, it means unacceptable delay imposed by employing single diagnostic services in a sequential manner is mitigated. Furthermore, although not directly studied in this paper, the DMDT can improve the efficiency of the weekly sarcoma MDT as cases have been stream-lined prior to being added to the discussion list, meaning greater cognitive bandwidth is available to discuss more complex patients.

\section{Limitations}

Our study was conducted in a high volume, tertiary centre for bone and soft tissue sarcoma. As a result, the population studied is niche and small meaning wider generalisability could be lost. Bone and soft tissue sarcomas are inherently rare conditions which, for the majority of clinicians, may never be encountered in everyday practice. There are 5 centres in England that provide diagnostic services for suspected bone sarcoma - we believe our data are demographically similar to that of the wider sarcoma population and could be extrapolated and applied to their populations despite the small sample size. One could argue that this methodology is also well suited to other image-based diagnostic conditions, eg, neurological, liver and lung cancers and since the number of referrals for these cancers is greater than that of sarcoma, the impact of frequent diagnostic MDTs (outside of formal cancer MDTs) may be larger.

Efforts have been made to reduce selection bias by selecting the same time period (January) for both cohorts. This was to mitigate the impact the time of year may have 
had on the number of cases triaged at our centre. Provider availability in 2015 may also have affected our results - in particular, time to first management decision. Our analysis could be strengthened by selecting two cohorts from different months to mitigate this. However, as we now provide an adequately staffed year-round service, we anticipate that this would have little impact on the findings.

Lastly, this study has not assessed the psychological impact and clinical outcomes of these patients; however, we anticipate that a faster triage process and an earlier diagnosis following the introduction of the DMDT has had a positive impact on these outcomes. Further studies are required to assess the impact of earlier diagnosis on longterm outcomes.

\section{Conclusion}

This pioneering measure of introducing a DMDT has multiple benefits. The daily diagnostic MDT has reduced our overall time to definitive diagnosis. The DMDT ensures all new referrals receive a prompt, consistent opinion, which can be fed back directly to the patients and referring clinicians. In particular, 24\% of patients were discharged without the need for them to travel to our department. As the April 2020 deadline for the introduction of the new cancer waiting times target comes into force, the ability to risk stratify and direct resources appropriately becomes ever more important. The implementation of this DMDT model requires the engagement and collaboration of the whole MDT and wider hospital management in order to achieve and embed these benefits. However, we propose that our DMDT is a reproducible model that can be employed in other cancer services involved in both the diagnosis and treatment of patients with the aim of reducing time to definitive diagnosis, directing resources, and improving the patient pathway.

\section{Data Sharing Statement}

The data that support the findings of this study are available from the corresponding author upon reasonable request.

\section{Acknowledgments}

This paper was presented at the following conferences with interim findings:

British Sarcoma Group Conference, 27-28 February 2019, London, UK - oral presentation.
British Orthopaedic Oncology Society Meeting, 4-5 April 2019, Leiden, The Netherlands - poster.

European Musculoskeletal Oncology Society Meeting, 14-16 May 2019, Florence, Italy - poster. British Orthopaedic Association Congress 10-13 September 2019, Liverpool, UK - poster.

International Society of Limb Salvage Meeting, 11-14 September 2019, Athens, Greece - poster.

British Association for Surgical Oncology Meeting, 16 November 2019, London, UK - poster.

British Orthopaedic Trainees Association Conference, 27-29 November 2019, Edinburgh, UK - poster.

Conference abstract published in the European Journal of Surgical Oncology, Volume 45, Issue 11, p2198, DOI: https://doi.org/10.1016/j.ejso.2019.09.017.

\section{Disclosure}

The authors report no conflicts of interest for this work.

\section{References}

1. Munro AJ. Multidisciplinary team meetings in cancer care: an idea whose time has gone? Clin Oncol. 2015;27:728-731. doi:10.1016/j. clon.2015.08.008

2. Calman K, Hine D. A policy framework for commissioning cancer services; a report by the Expert Advisory Group on Cancer to the Chief Medical Officers of England and Wales; 1995.

3. Ruhstaller T, Roe H, Thürlimann B, et al. The MDT: an indispensable aid to communication between different specialties. Eur $J$ Cancer. 2006;42(15):2459-2462. doi:10.1016/j.ejca.2006.03.034

4. Taylor C, et al. Multidisciplinary team working in cancer; what is the evidence? BMJ. 2010;340:c951.

5. Cancer Waiting Times Annual Report 2015/16 p4 section 1.2 table 1.1 Published to the NHS England website, in electronic PDF format only. Available from: http://www.england.nhs.uk/statistics/statisticalwork-areas/. Accessed December 30, 2020.

6. Cancer Waiting Times Annual Report 2016/17 p4 section 1.2 table 1.1 Published to the NHS England website, in electronic PDF format only. Available from: http://www.england.nhs.uk/statistics/statisticalwork-areas/. Accessed December 30, 2020.

7. Cancer Waiting Times Annual Report 2017/18 p4 section 1.2 table 1.1 Published to the NHS England website, in electronic PDF format only. Available from: http://www.england.nhs.uk/statistics/statisticalwork-areas/. Accessed December 30, 2020.

8. Lacobucci G. Patients left waiting for cancer treatment as targets missed, BMA analysis shows. BMJ. 2019;365:I2039. doi:10.1136/bmj.12039

9. De Iso PB, Coward JI, Letsa I, et al. A study of the decision outcomes and financial costs of multidisciplinary team meetings (MDMs) in oncology. $B r \quad J$ Cancer. 2013;109(9):2295-2300. doi:10.1038/bjc.2013.586

10. Fleissig A, Jenkins V, Catt S, et al. Multidisciplinary teams in cancer care: are they effective in the UK? Lancet Oncol. 2006;7 (11):935-943. doi:10.1016/S1470-2045(06)70940-8

11. Cancer Research UK 'meeting patients' needs: improving the effectiveness of multidisciplinary team meetings' January 2017. Published to the Cancer Research UK website, in electronic PDF format only. Available from: https://www.cancerresearchuk.org/about-us/we-develop-policy /our-policy-on-cancer-services/improving-the-effectiveness-of-mdts-incancer-services. Accessed December 30, 2020. 
12. Chinai N, Bintcliffe F, Armstrong EM, et al. Does every patient need to be discussed at a multidisciplinary team meeting? Clin Radiol. 2013;68(8):780-784. doi:10.1016/j.crad.2013.02.011

13. Jalil R, Ahmed M, Green JSA, et al. Factors that can make an impact on decision-making and decision implementation in cancer multidisciplinary teams: an interview study of the provider perspective. Int J Surg. 2013;11(5):389-394. doi:10.1016/j.ijsu.2013.02.026

14. Transforming multidisciplinary team meetings. Martin Gore; 2017

15. "Achieving world-class cancer outcomes: a strategy for England" section 5.5.1. The Independent Cancer Taskforce; July 2015

16. Dangoor A, Seddon B, Gerrand C, et al. UK guidelines for the management of soft tissue sarcomas. Clin Sarcoma Res. 2016;6:20. doi:10.1186/s13569-016-0060-4

17. Gerrand C, Athanasou N, Brennan B, et al. UK guidelines for the management of bone sarcomas. Clin Sarcoma Res. 2016;6:7. doi:10.1186/s13569-016-0047-1

18. Grimer R, et al. UK guidelines for the management of bone sarcomas. Sarcoma. 2010;2010:317462.

19. West Midlands Cancer Intelligence Unit. Bone and soft tissue sarcomas UK incidence and survival: 1996 to 2010; 2013.

20. National Cancer Registration and Analysis Service. Soft tissue sarcomas: incidence and survival rates in England; 2011.

21. Boo SL, Botchu R, McLoughlin E, et al. Commentary: sarcoma multidisciplinary team meeting, past, present and future. Clin Radiol. 2020;75:316-318. doi:10.1016/j.crad.2019.12.015

22. Hiom SC. Diagnosing cancer earlier: reviewing the evidence for improving cancer survival. $B r \quad J$ Cancer. 2015;112(1):S1-5. doi:10.1038/bjc.2015.23

23. Pencavel TD, Strauss DC, Thomas GP, et al. Does the two-week rule pathway improve the diagnosis of soft tissue sarcoma? A retrospective review of referral patterns and outcomes over five years in a regional sarcoma centre. Ann R Coll Surg Engl. 2010;92 (5):417-4211. doi:10.1308/003588410X12664192075972
24. Chang AE, et al. Multidisciplinary cancer clinics: their time has come. J Surg Oncol. 1998;69:203-205. doi:10.1002/(SICI)10969098(199812)69:4<203::AID-JSO2>3.0.CO;2-Z

25. Smith GM, Johnson GD, Grimer RJ, et al. Trends in presentation of bone and soft tissue sarcomas over 25 years: little evidence of earlier diagnosis. Ann R Coll Surg Engl. 2011;93(7):542-547. doi:10.1308/ 147870811X13137608455055

26. Bauer HC, Trovik CS, Alvegård TA, et al. Monitoring referral and treatment in soft tissue sarcoma: study based on 1851 patients from the Scandinavian Sarcoma Group Register. Acta Orthop Scand. 2001;72:150-159. doi:10.1080/000164701317323408

27. Di Girolamo C, Walters S, Gildea C, et al. Can we assess Cancer Waiting Time targets with cancer survival? A population-based study of individually linked data from the National Cancer Waiting Times monitoring dataset in England, 2009-2013. PLoS One. 2018;13(8): e0201288. doi:10.1371/journal.pone.0201288

28. Brocken P, Prins JB, Dekhuijzen PNR, et al. The faster the better? A systematic review on distress in the diagnostic phase of suspected cancer, and the influence of rapid diagnostic pathways. Psychooncology. 2012;21(1):1-10. doi:10.1002/pon.1929

29. Saegrov S, Halding A-G. What is it like living with a diagnosis of cancer? Eur J Cancer Care (Engl). 2004;13(2):145-153. doi:10.1111/ j.1365-2354.2004.00442.x

30. Scott CEH, MacDonald DJ, Howie CR, et al. 'Worse than death' waiting for a joint arthroplasty. Bone Joint J. 2019;101-B (8):941-950. doi:10.1302/0301-620X.101B8.BJJ-2019-0116.R1

31. Chaudhry SR, Tsetse C, Chennan SE, et al. Early recognition and diagnosis of Ewing Sarcoma of the cervical spine. Radiol Case Rep. 2019;14(2):160-163. doi:10.1016/j.radcr.2018.09.017

32. Loud J, Murphy J. Cancer screening and early detection in the 21 st century. Semin Oncol Nurs. 2018;33(2):121-128. doi:10.1016/j. soncn.2017.02.002
Journal of Multidisciplinary Healthcare

\section{Publish your work in this journal}

The Journal of Multidisciplinary Healthcare is an international, peerreviewed open-access journal that aims to represent and publish research in healthcare areas delivered by practitioners of different disciplines. This includes studies and reviews conducted by multidisciplinary teams as well as research which evaluates the results or conduct of such teams or healthcare processes in general. The journal covers a very wide range of areas and welcomes submissions from practitioners at all levels, from all over the world. The manuscript management system is completely online and includes a very quick and fair peer-review system. Visit http://www.dovepress.com/testimonials. php to read real quotes from published authors. 